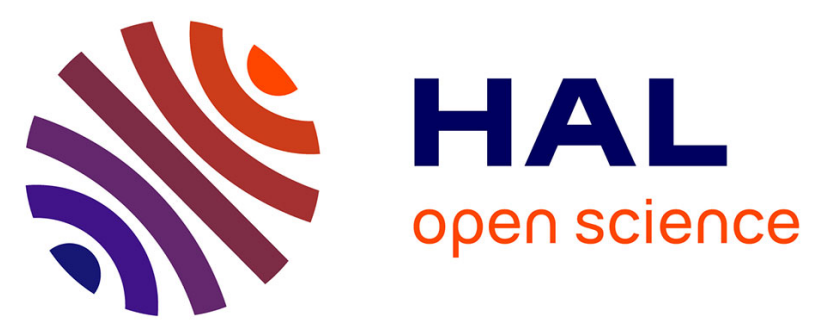

\title{
Qualité nutritionnelle, microbiologique et stabilité à l'oxydation de formules infantiles fortifiées ou aliments thérapeutiques dans les pays du sud
}

Célia Moustiès, Claire Bourlieu, Bruno Barea, Adrien Servent, Youna Hémery, Sylvie Avallone

\section{To cite this version:}

Célia Moustiès, Claire Bourlieu, Bruno Barea, Adrien Servent, Youna Hémery, et al.. Qualité nutritionnelle, microbiologique et stabilité à l'oxydation de formules infantiles fortifiées ou aliments thérapeutiques dans les pays du sud. Les Journées Francophones de nutrition JFN 2017, Dec 2017, Nantes, France. 2017. hal-01837518

\author{
HAL Id: hal-01837518 \\ https://hal.science/hal-01837518
}

Submitted on 2 Jun 2020

HAL is a multi-disciplinary open access archive for the deposit and dissemination of scientific research documents, whether they are published or not. The documents may come from teaching and research institutions in France or abroad, or from public or private research centers.
L'archive ouverte pluridisciplinaire HAL, est destinée au dépôt et à la diffusion de documents scientifiques de niveau recherche, publiés ou non, émanant des établissements d'enseignement et de recherche français ou étrangers, des laboratoires publics ou privés. 
Qualité nutritionnelle, microbiologique et stabilité à l'oxydation de formules infantiles fortifiées ou aliments thérapeutiques dans les pays du sud

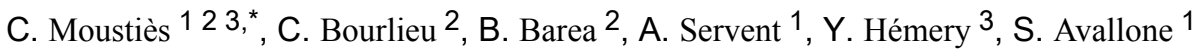 \\ 1'UMR 95 QUALISUD, SUPAGRO/CIRAD , 2UMR 1208 IATE, INRA/CIRAD , ${ }^{3}$ UMR 204 \\ NUTRIPASS, IRD, Montpellier, France
}

Introduction et but de l'étude: L'alimentation infantile est un enjeu dans les pays du Sud car la malnutrition touche encore 200 millions d'enfants âgés de moins de 5 ans (UNICEF, WHO, 2016). Les causes de cette malnutrition peuvent être liées à une ration alimentaire inadéquate (apports insuffisants en micronutriments) associée ou non à une ou des maladies (UNICEF, 1997). Afin de palier à cette malnutrition, diverses stratégies sont mises en place, notamment l'enrichissement/fortification d'aliments utilisés en complément de l'allaitement maternel ou en soin thérapeutique de type farines infantiles fortifiées et aliments thérapeutiques prêts à l'emploi. L'objectif de cette étude est d'évaluer la qualité nutritionnelle, microbiologique ainsi que la stabilité à l'oxydation des vitamines liposolubles et des lipides dans ces deux types de matrices prélevées dans les circuits de distribution des pays du Sud.

Matériel et méthodes: Un panel de 13 farines infantiles (produites par des entreprises internationales, des ONGs, ou de petites entreprises locales africaines ou asiatiques) et trois aliments thérapeutiques prêts à l'emploi ont été prélevés dans les pays de distribution et analysés à différents temps après leur date de fabrication.

Les vitamines liposolubles (A, D, E) ont été dosées par chromatographie en phase liquide haute pression, les composés secondaires de dégradation par chromatographie en phase gazeuse. Après extraction Folch, la fraction lipidique a été analysée en terme d'acides gras totaux, classes de lipides, indices de peroxyde (IP) et d'acide. Enfin, les microorganismes aérobies mésophiles, entérobactéries, salmonelles, cronobacter sp., et les mycotoxines totales ont été recherchés dans les matrices.

Résultats et Analyse statistique : La majorité des farines infantiles présentent des teneurs en vitamines $\mathrm{A}$ et $\mathrm{D}$ au-dessus des normes ISO, mais en dessous de la norme ISO pour la vitamine $\mathrm{E}$. Quelques matrices ont des teneurs bien en deçà des normes. La fraction lipidique est composée en majorité de triglycérides, avec pour principaux acides gras les C16:0, C18:1 et C18:2. Le ratio $\omega 6 / \omega 3$ est très satisfaisant dans le cas des aliments thérapeutiques prêts à l'emploi (moyenne=3.6), mais n'est

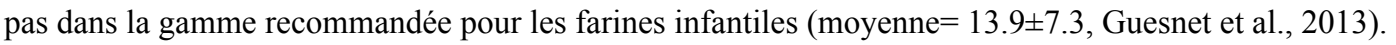
L'état d'oxydation de certaines matrices est avancé, avec des IP dépassant le seuil autorisé (IPmax 10 $\mathrm{mq} \mathrm{O}_{2} / \mathrm{Kg}$ ). Au niveau microbiologique, la moitié des matrices analysées sont non satisfaisantes.

Globalement, que ce soit sur le plan nutritionnel ou microbiologique, les farines infantiles produites par des entreprises internationales ne présentent pas un meilleur profil que celles produites par les petites entreprises locales.

Conclusion: La durée de stockage, la formulation ainsi que l'emballage impactent la stabilité des vitamines, et l'état d'oxydation des matrices fortifiées, dont certaines présentent des profils nutritionnels et/ou microbiologiques non satisfaisants, bien avant la date limite de consommation spécifiée.

Remerciements: Le Groupe Lipides Nutrition a soutenu ce travail par l'attribution d'une bourse.

\title{
Conflits d'intérêts: Aucun conflit à déclarer
}

